This item is the archived peer-reviewed author-version of:

\title{
A first-principles study of stable few-layer penta-silicene
}

\section{Reference:}

Aierken Yierpan, Leenaerts Ortwin, Peeters François.- A first-principles study of stable few-layer penta-silicene Physical chemistry, chemical physics / Royal Society of Chemistry [London] - ISSN 1463-9076 - 18:27(2016), p. 18486-18492 Full text (Publisher's DOI): http://dx.doi.org/doi:10.1039/C6CP03200A

To cite this reference: http://hdl.handle.net/10067/1349420151162165141 

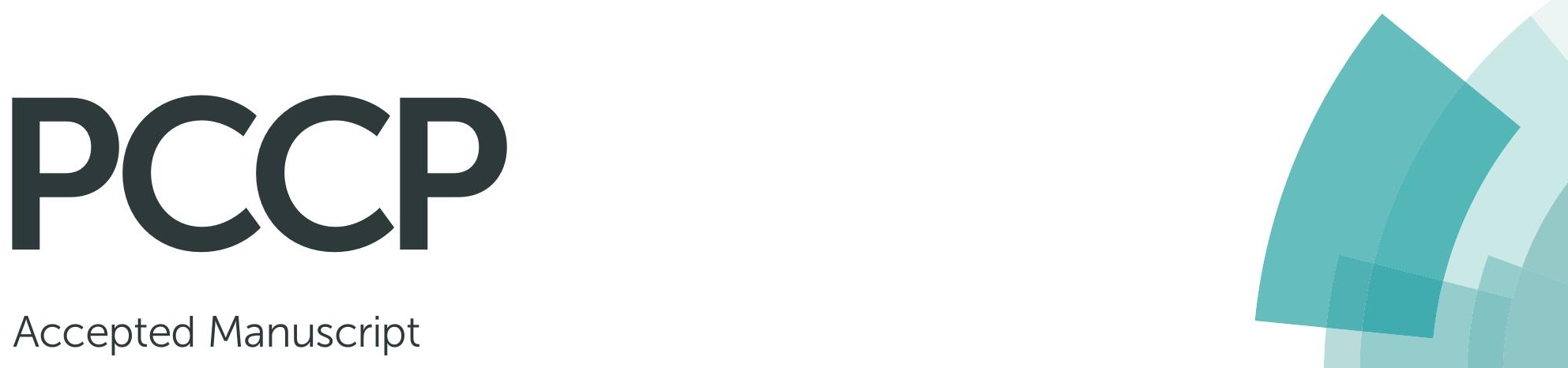

\section{Accepted Manuscript}

This article can be cited before page numbers have been issued, to do this please use: Y. Aierken, $\mathrm{O}$. Leenaerts and F. Peeters, Phys. Chem. Chem. Phys., 2016, DOI: 10.1039/C6CP03200A.

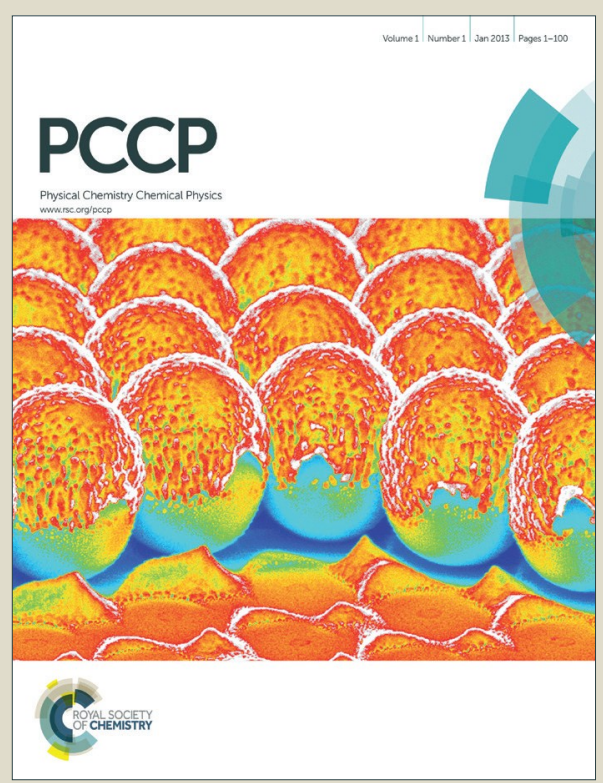

This is an Accepted Manuscript, which has been through the Royal Society of Chemistry peer review process and has been accepted for publication.

Accepted Manuscripts are published online shortly after acceptance, before technical editing, formatting and proof reading. Using this free service, authors can make their results available to the community, in citable form, before we publish the edited article. We will replace this Accepted Manuscript with the edited and formatted Advance Article as soon as it is available.

You can find more information about Accepted Manuscripts in the Information for Authors.

Please note that technical editing may introduce minor changes to the text and/or graphics, which may alter content. The journal's standard Terms \& Conditions and the Ethical guidelines still apply. In no event shall the Royal Society of Chemistry be held responsible for any errors or omissions in this Accepted Manuscript or any consequences arising from the use of any information it contains. 


\section{Journal Name}

\section{ARTICLE TYPE}

Cite this: DOI: $10.1039 / x x x x x x x x x x$

\section{First-principles study of stable few-layer penta- silicene ${ }^{\dagger}$}

\author{
Yierpan Aierken, ${ }^{a}$ Ortwin Leenaerts ${ }^{b}$, and François M. Peeters ${ }^{c *}$
}

Received Date

Accepted Date

DOI: 10.1039/xxxxxxxxxx

www.rsc.org/journalname

\begin{abstract}
Recently penta-graphene was proposed as a stable two-dimensional carbon allotrope consisting of a single layer of interconnected carbon pentagons [Zhang et al., PNAS, 2015, 112, 2372]. Its silicon counterpart, penta-silicene, however, is not stable. In this work, we show that multilayers of penta-silicene form stable materials with semiconducting or metallic properties, depending on the stacking mode. We demonstrate their dynamic stability through their phonon spectrum and using molecular dynamics. A particular type of bilayer penta-silicene is found to have lower energy than all of the known hexagonal silicene bilayers and forms therefore the most stable bilayer silicon material predicted so far. The electronic and mechanical properties of these new silicon allotropes are studied in detail and their behavior under the strain is investigated. We demonstrate that strain can be used to tune its band gap.
\end{abstract}

\section{Introduction}

It has been more than a decade since the discovery of graphene ${ }^{1-3}$, a surprisingly stable two-dimensional (2D) carbon allotrope. Driven by the extraordinary properties of this pioneer material ${ }^{3-6}$, such as high carrier mobility ${ }^{7}$ and mechanical strength ${ }^{8}$, the research community on two-dimensional crystals is expanding with unprecedented speed. A great number of graphene-like structures and other layered structures with different chemical compositions and different crystal structures have been proposed and synthesized ${ }^{9-11}$. Recently, a new 2D structure for carbon was proposed, called penta-graphene ${ }^{12}$. This crystal is composed entirely of pentagonal rings of $\mathrm{C}$ atoms with mixed $\mathrm{sp}^{2} / \mathrm{sp}^{3}$ orbital hybridization. However, the silicon counterpart of this structure, penta-silicene, contains a dynamical instability in its monolayer form. A few attempts have been made to stabilize this new Si structure by hydrogenation ${ }^{13}$ and chemical doping ${ }^{14}$.

In the present work, we construct multilayer structures of penta-silicene. We use density functional theory to explore their stability and physical properties. Two types of stacking for the penta-silicene layers are found to give stable few-layer structures. These different stacking types lead to completely different electronic properties since one leads to metallic and the other to

Department of Physics, University of Antwerp, Groenenborgerlaan 171, 2020 Antwerpen, Belgium. Fax: +32 326535 42; Tel: +3232653664.

${ }^{a}$ E-mail: yierpan.aierken@uantwerpen.be

${ }^{b}$ E-mail: ortwin.leenaerts@uantwerpen.be

${ }^{c}$ E-mail: francois.peeters@uantwerpen.be

$\dagger$ Electronic Supplementary Information (ESI) available: [The structure and physical properties of h-Sil, and the structure files of all newly purposed systems]. See DOI: $10.1039 / \mathrm{b} 000000 \mathrm{x} /$ semiconducting behavior. Somewhat surprisingly, we found that bilayer penta-silicene has lower formation energy than the most stable hexagonal silicene bilayers. Furthermore, we found that the band gaps of these semiconducting penta-silicene bilayers can be tuned by mechanical strain.

Our work is organized as follows: In Sec. 3.1, we explore the stability of monolayer penta-silicene and demonstrate its dynamical instability. This forms the motivation to study few-layer systems. We investigate different stacking possibilities and the resulting stability in Sec. 3.2 and Sec. 3.3, respectively. Additionally, we study their mechanical properties by calculating their elastic constants. In Sec. 3.4, we compare bilayer penta-silicene to the most stable bilayer hexagonal silicene structures. The electronic properties of multilayered penta-silicene are discussed in Sec. 3.5 together with the influence of strain on the band gap. Final conclusions are presented in Sec. 4.

\section{Computational details}

To calculate the ground state properties of our systems, we use first-principles methods in the framework of density functional theory (DFT) as implemented in the Vienna ab initio Simulation Package (VASP). ${ }^{15-18}$ VASP makes use of a plane-wave basis set and the projected augmented wave method ${ }^{19,20}$ to describe the wave functions. The plane wave cut-off energy is chosen to be $500 \mathrm{eV}$. The exchange-correlation potential is treated with the generalized gradient approximation (GGA) within the PerdewBurke-Ernzerhof (PBE) formulation ${ }^{21,22}$. To make sure GGA-PBE has qualitatively consistent description with the one includes van der Waals (vdW) correction, we have compare them in the final results. The vdW is included through DFT-D3(BJ) method from 
Grimme et al. ${ }^{23}$ together with Becke-Jonson (BJ) damping ${ }^{24}$. A $11 \times 11 \times 1$ k-points mesh is used to sample the Brillouin zone for geometry relaxation. Finer meshes are used for total energy calculations: a $17 \times 17 \times 1$ grid for insulating and a $23 \times 23 \times 1$ grid for metallic slabs. For bulk systems, a similar k-point density is used for the normal direction as for the $2 \mathrm{D}$ plane, i.e. depending on the inverse length of the $\mathbf{c}$ lattice vector. The separation of (artificial) periodic images due to the periodic boundary conditions is set to at least $20 \AA$. Phonon spectra are evaluated with the finite-difference method from VASP together with the Phonopy program ${ }^{25}$. To obtain accurate forces, supercells are generated for the phonon calculations: $4 \times 4 \times 1$ and $3 \times 3 \times 2$ supercells are used for few-layer and bulk systems, respectively. The self-consistent convergence criterion is set to $10^{-8} \mathrm{eV}$ for the energy, while forces are relaxed below $10^{-7} \mathrm{eV} / \mathrm{A}$. Ab initio molecular dynamics is performed with Parrinello-Rahman (NpT) dynamics $^{26,27}$ and a Langevin thermostat ${ }^{28}$ as implemented in VASP. In these simulations, the plane wave cut-off energy is lowered to $300 \mathrm{eV}$. The temperature is set to $100 \mathrm{~K}$ and a time step of $2 \mathrm{fs}$ is used to obtain a total simulation time of $6 \mathrm{ps}$.

\section{Results}

\subsection{Monolayer penta-silicene}

The layer group symmetry of monolayer penta-silicene is $\mathrm{p} \overline{4} 2_{1} \mathrm{~m}$ (58). As shown in Fig. 1(a) and Fig. 1(b), the primitive cell contains six silicon atoms, of which two have fourfold coordination (Si4) and four have threefold coordination (Si3). Two of the Si3 atoms reside above the $\mathrm{Si} 4$ atoms, denoted as $\mathrm{Si3}$ _u, while the other two are below the Si4 atoms, denoted as Si3_d. The Si4 atoms are bonded to four $\mathrm{Si} 3$ atoms while the $\mathrm{Si} 3$ atoms are connected to two Si4 atoms and one neighboring Si3 atom. Note that the two Si4 atoms have equivalent environments which are rotated by approximately $41^{\circ}$ with respect to each other. Therefore, in analogy to graphene, we can relate these two equivalent $\mathrm{Si} 4$ atoms to sublattices which in the following will be referred to as the A and B sublattice.

The dynamical stability of this structure can be studied through its phonon spectrum. As noted before ${ }^{13,14}$ the phonon spectrum of monolayer penta-silicene contains imaginary frequencies as shown in Fig. 1(c), which is a clear signature of its instability. The corresponding atomic vibrations of the two imaginary frequencies at the $\Gamma$ point are shown in Fig. 1(d). These modes correspond mainly to out-of-plane vibrations of the $\mathrm{Si3}$ atoms with respect to the Si4 atoms. As a consequence, the structure is found to fall apart, indicating that there is no stable form of monolayer pentasilicene. However, the addition of extra layers could reduce these out of plane vibrations and stabilize the structure. This is the motivation to study few-layer penta-silicene.

\subsection{Multilayers of penta-silicene structures}

When considering two layers, different stacking configurations are possible. Here we focus on the so-called AA and AB stacking modes of the aforementioned sublattices (see Fig. 2). The stacking in which both layers have the same in-plane orientation and the Si atoms are put right on top of each other is called AA stack- (a)

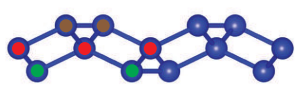

$\mathrm{Si3}$ d $\bullet \mathrm{Si3}$ u $\bullet \mathrm{Si} 4 \bullet$

(c)

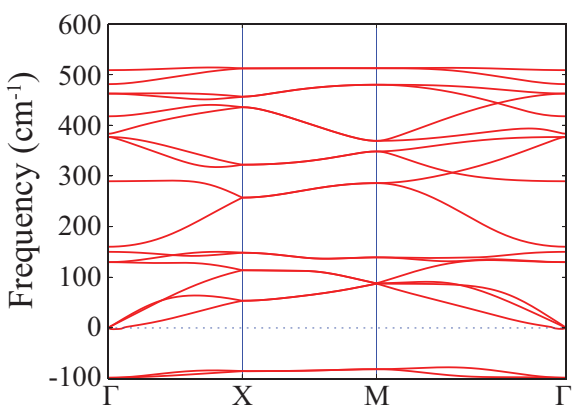

(d)

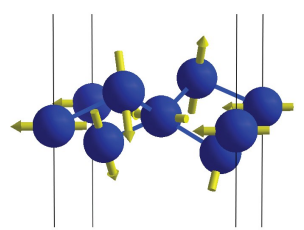

(b)
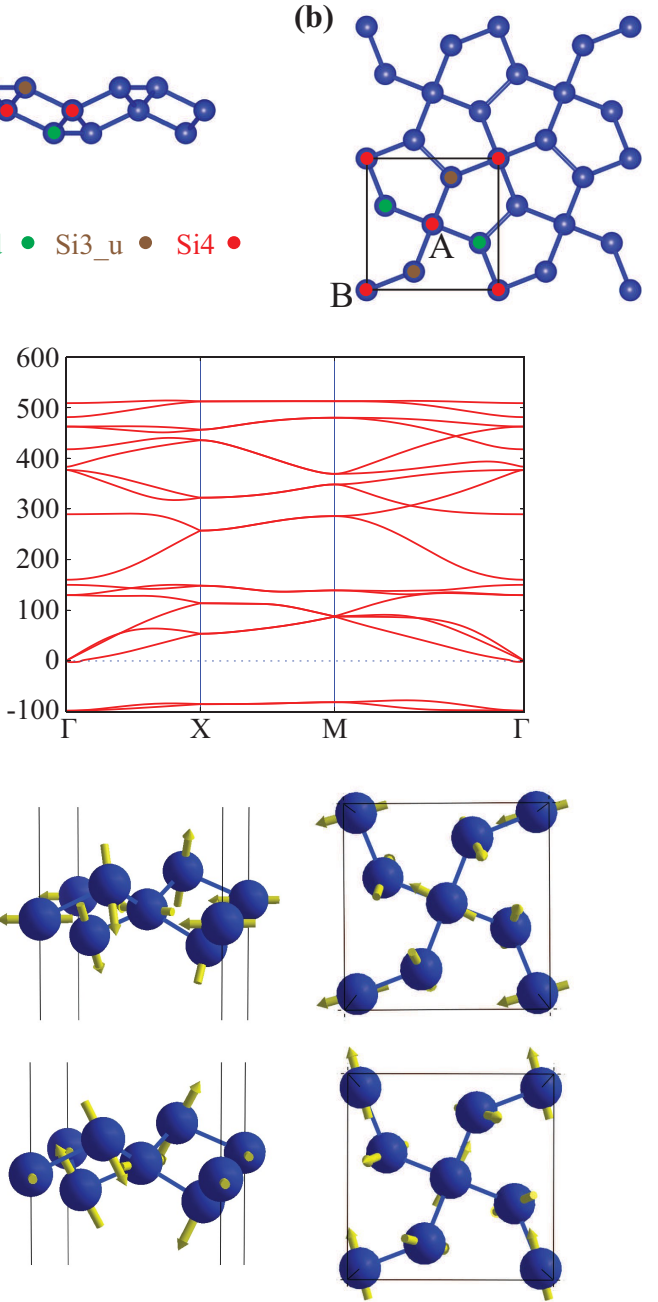

Fig. 1 (a) Side view and (b) top view of the atomic structure, (c) phonon spectrum and (d) two vibration modes with imaginary frequency of monolayer penta-silicene. Visualization of vibration modes is done with the V_Sim package ${ }^{29}$.
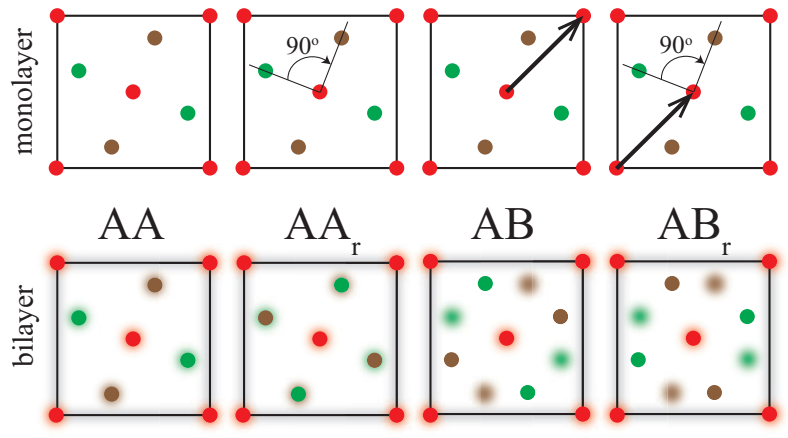

Fig. 2 Schematic illustration of the four stacking types for bilayer pentasilicene. The colors of the symbols correspond to those of the monolayer in Fig. 1(a) and Fig. 1(b). The bottom layer in the bilayer is blurred for clarity. The arrow represents translation and the angle represents the rotation of the top layer with respect to the bottom layer. 
Table 1 The cohesive energy $\left(E_{\text {coh }}\right)$, the interlayer binding distance $\left(d_{\text {inter }}\right)$, the interlayer binding energy $\left(E_{\text {inter }}\right)$, number of interlayer bonds $\left(N_{b}\right)$ and energy per bond ( $E_{\text {bond }}$ ) of the four possible stacking types of bilayer penta-silicene. The interlayer binding energy per unit cell is defined as $E_{\mathrm{inter}}=E_{\mathrm{bi}}-2 E_{\mathrm{mono}}$.

\begin{tabular}{cccccc}
\hline structure & $\begin{array}{c}\mathrm{E}_{\text {coh }} \\
(\mathrm{eV} / \text { atom })\end{array}$ & $\begin{array}{c}\mathrm{d}_{\text {inter }} \\
(\AA)\end{array}$ & $\begin{array}{c}\mathrm{E}_{\text {inter }} \\
(\mathrm{eV})\end{array}$ & $\begin{array}{c}\mathrm{N}_{b} \\
-\end{array}$ & $\begin{array}{c}\mathrm{E}_{\text {bond }} \\
(\mathrm{eV})\end{array}$ \\
\hline $\mathrm{AA}$ & -4.129 & 0.795 & -3.502 & 4 & -0.875 \\
$\mathrm{AA}_{r}$ & -4.113 & 2.379 & -3.318 & 2 & -1.659 \\
$\mathrm{AB}$ & -3.968 & 2.174 & -1.574 & 2 & -0.787 \\
$\mathrm{AB}_{r}$ & -4.147 & 1.893 & -3.725 & 2 & -1.862 \\
$\mathrm{AB}_{r}^{d}$ & -4.185 & 1.896 & -4.174 & 2 & -2.087 \\
\hline
\end{tabular}

(1) bilayer

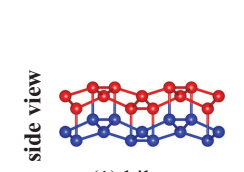

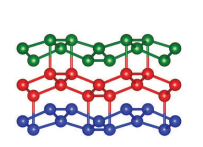

(2) trilayer

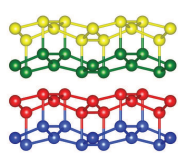

(3) tetralayer

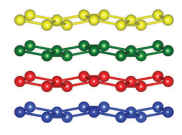

(4) bulk (a) AA stacked structures
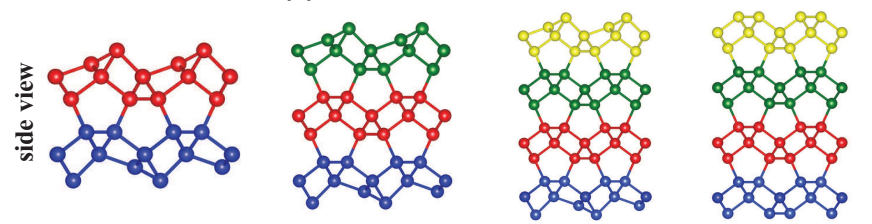

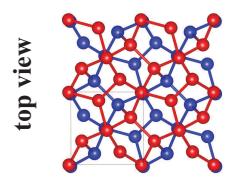

(1) bilayer

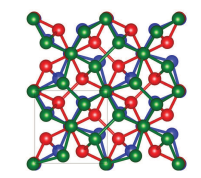

(2) trilayer

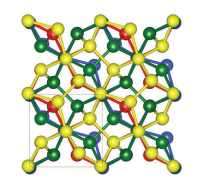

(3) tetralayer

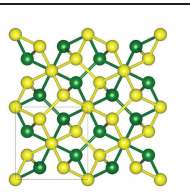

(4) bulk (b) $\mathrm{AB}_{r}^{d}$ stacked few-layer and $\mathrm{AB}_{r}$ stacked bulk structures

Fig. 3 Atomic structure of the $2 \times 2$ supercell of few-layer penta-silicene. The number of atomic layers in the bulk structure is fixed to four for comparison (i.e. $2 \times 2 \times 4$ and $2 \times 2 \times 2$ supercells for $A A$ and $\mathrm{AB}_{r}$ stacked bulk penta-silicene). (Visualisation using VESTA ${ }^{30}$ ).

ing. $A B$ stacking arises by shifting the A sublattice of one layer to the B sublattice of the other. This nomenclature was also used by Wang et al. ${ }^{31}$ for bilayer penta-graphene. Although pentasilicene has a tertragonal lattice symmetry, the highest proper rotational symmetry order is two. Therefore, there are also two different possible orientations of the upper layer with respect to the lower one: One in which the two layers have the same orientation and another in which one layer is rotated over $90^{\circ}$ with respect to the other one. We denote this last orientation with a subscript $r$ to show that it results from a $90^{\circ}$ rotation, e.g. $\mathrm{AA}_{r}$. Therefore, there are four possible stacking types for bilayer penta-silicene. Note that $\mathrm{AB}_{r}$ stacking corresponds to the recently proposed bulk T12 phase for group IVA elements ${ }^{32}$. However, as discussed in more detail below, perfect $\mathrm{AB}_{r}$ stacking is not stable in the case of multilayer penta-silicene. A considerable distortion of the outer layers is required to stabilize $\mathrm{AB}_{r}$ stacking. The distorted structure, which will be referred to as $\mathrm{AB}_{r}^{d}$ in the following, is obtained by breaking the symmetry between the two $\mathrm{Si} 3$ atoms at each surface side in $\mathrm{AB}_{r}$ multilayers. In this way, one of the two Si3 atoms acquires $\mathrm{sp}^{2}$ hybridization and looses an electron to the other $\mathrm{Si} 3$ atom that has $\mathrm{sp}^{3}$ hybridization.

In Table 1, we compare the energies of the different stacking modes. In all cases the Si4 atoms are not involved in interlayer bonding since their possible number of bonds is already saturated. Except for the AA stacking where all Si3 atoms are bound to Si3 atoms from the other layer, only half of the $\mathrm{Si} 3$ atoms are bonded to the other layer in the other cases. In Table 1 , the size of the interlayer binding energy and the strength per bond are given. The size of the bond energies indicate strong chemical bonding. The $\mathrm{AB}_{r}^{d}$ stacking mode clearly forms the most stable structure. For the rest of the paper, we will only focus on the most stable AA and $\mathrm{AB}$-type stacking, i.e. $\mathrm{AA}$ and $\mathrm{AB}_{r}^{d}$.

We also investigated the stability of trilayer, tetralayer and bulk penta-silicene structures by adding extra layers to the stable bilayers mentioned above, their structures are shown in Fig. 3(a) and Fig. 3(b). We list their structural and energetic properties in Table 2. Extra layers increase the cohesive energy per atom due to a smaller ratio of surface atoms. For AA stacking, adding a 4th layer to a trilayer system results in a double bilayer system with lesser bonding between them. Going to AA bulk, the interlayer interaction appears to be further reduced and the buckled layers become more flat. Adding extra layers to an $\mathrm{AB}_{r}^{d}$ bilayer results in similar structures in which the $\mathrm{Si} 3$ atoms of the surface layers become distorted. For bulk, the undistorted $\mathrm{AB}_{r}$ structure is found in which $\overline{4}$-fold symmetry is restored.

\subsection{Stabilities}
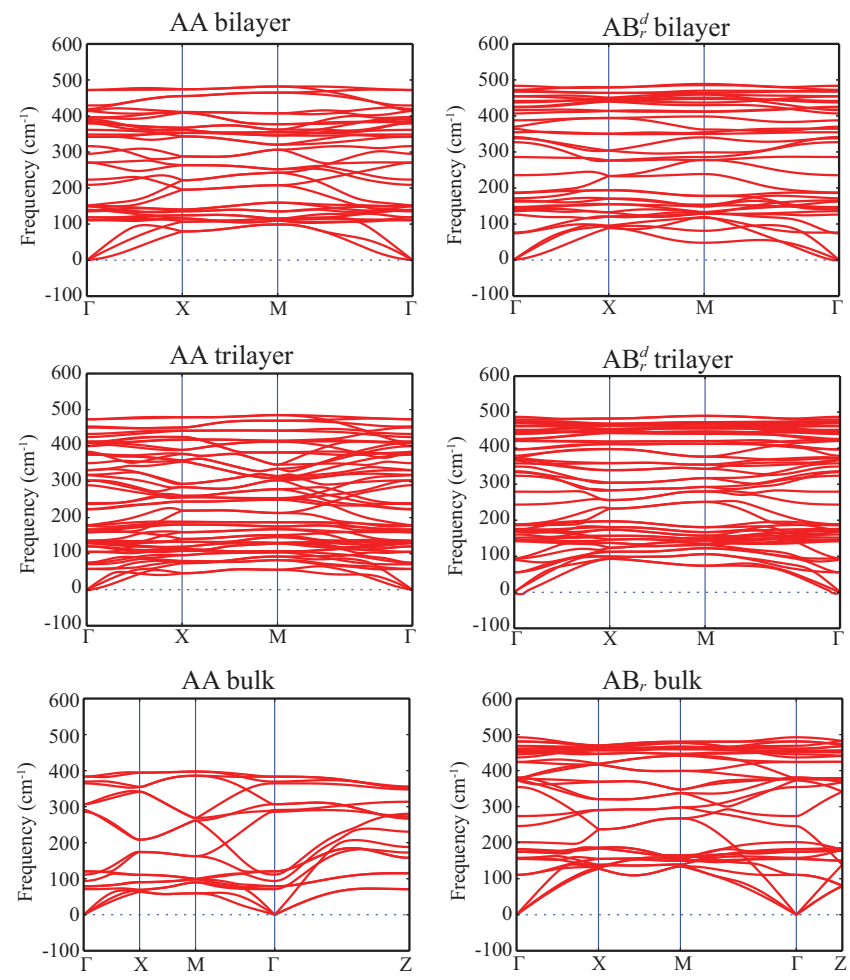

Fig. 4 Phonon spectra of different-stacked few-layer penta-silicene.

In this section we investigate the stability of the different multi- 
Table 2 The layer (space) group for few-layer (bulk) systems. The lattice constant $(a)$, the interlayer distance ( $\mathrm{d}_{\text {inter }}$ ), the nearest-neighbor bond length range $\left(d_{\min / \max }\right)$, the cohesive energy $\left(E_{c o h}\right)$, and the band gap (PBE) of few-layer and bulk penta-silicene.

\begin{tabular}{|c|c|c|c|c|c|c|c|c|}
\hline stacking & structure & layer/space group & $a(\AA)$ & $\mathrm{d}_{\text {inter }}(\AA)$ & $\mathrm{d}_{\min }(\AA)$ & $\mathrm{d}_{\max }(\AA)$ & $\mathrm{E}_{\mathrm{coh}}(\mathrm{eV} /$ atom $)$ & band gap (eV) \\
\hline- & monolayer & $\mathrm{p} \overline{4} 2_{1} \mathrm{~m}(58)$ & 5.587 & - & 2.233 & 2.363 & -3.837 & $0.046(\mathrm{M} \rightarrow \Sigma)$ \\
\hline \multirow{4}{*}{ AA } & bilayer & \multirow{3}{*}{$\mathrm{p} \overline{4} 2{ }_{1} \mathrm{~m}(58)$} & 5.907 & 0.795 & 2.363 & 2.468 & -4.129 & metal \\
\hline & trilayer & & 5.887 & 1.085 & 2.330 & 2.606 & -4.108 & metal \\
\hline & tetralayer & & 5.980 & $0.996 / 1.794^{\mathrm{a}}$ & 2.368 & 2.478 & -4.150 & metal \\
\hline & bulk & $\mathrm{P} \overline{4} 2_{1} \mathrm{~m}(113)$ & 6.234 & 1.769 & 2.398 & 2.463 & -4.204 & metal \\
\hline \multirow{3}{*}{$\mathrm{AB}_{r}^{d}$} & bilayer & pb2b (30) pm2a (31) & 5.222 & 1.896 & 2.303 & 2.403 & -4.185 & $0.119(\mathrm{M} \rightarrow \Sigma)$ \\
\hline & trilayer & $\mathrm{p} 1(1)$ & 5.222 & 1.989 & 2.298 & 2.413 & -4.291 & $0.247(\mathrm{M} \rightarrow \Sigma)$ \\
\hline & tetralayer & pb2b (30) pm2a (31) & 5.221 & 1.997 & 2.298 & 2.413 & -4.345 & $0.232(\mathrm{M} \rightarrow \Sigma)$ \\
\hline $\mathrm{AB}_{r}$ & bulk & $\mathrm{P}_{2} / \mathrm{ncm}(138)$ & 5.220 & 1.999 & 2.358 & 2.413 & -4.508 & $1.329(\mathrm{M} \rightarrow \Delta)$ \\
\hline
\end{tabular}

a The first and the second number indicate the interlayer distance between two monolayers and two bilayers, respectively.

layer structures discussed above. Phonon calculations for the AA and $\mathrm{AB}_{r}$ stacking modes reveal that only the AA bilayer is dynamically stable at low temperature. The extra bonds of the Si3 atoms in AA-stacked structures effectively reduce out-of-plane vibrations and stabilize the structure. Although the AA-stacked bulk structure has weaker interlayer bonding, its phonon spectrum contains no imaginary frequencies, indicating its dynamical stability. The $\mathrm{AB}_{r}$-stacked layers, on the other hand, exhibit similar out-of-plane vibrations of the outermost $\mathrm{Si}$ atoms as a monolayer. For $\mathrm{AB}_{r}^{d}$ stacking the distortion of the outermost layer removes the instabilities from the phonon spectrum, so that these structures are also dynamically stable.

It is also interesting to see whether these structures remain stable at finite temperature. To this end, we performed $a b$ initio molecular dynamics calculations at a temperature of $100 \mathrm{~K}$. The evolution of the cohesive energy as a function of simulation time is shown in Fig. 5. For comparison, the results for the dynamically unstable monolayer are also shown. The monolayer laterally shrinks and becomes a disordered multilayered system. The AA and $\mathrm{AB}_{r}^{d}$ bilayer systems, on the other hand, remain stable and retain their crystalline structure.

As a final stability check, we investigate the mechanical stability of bilayer penta-silicene which is determined by the elastic constants of the structures. If the elastic constants satisfy the necessary and sufficient Born criteria generalized by Mouhat and Coudert $^{33}$, the structures are mechanically stable. AA bilayer pentasilicene belong to the layer group symmetry of $\mathrm{p} \overline{4} 2_{1} \mathrm{~m}$, which belongs to the tetragonal symmetry groups, and the independent elastic constants in 2D are: $C_{11}=101.43 \mathrm{~N} / \mathrm{m}, C_{12}=36.36 \mathrm{~N} / \mathrm{m}$ and $C_{66}=39.53 \mathrm{~N} / \mathrm{m}$. In the case of $\mathrm{AB}_{r}^{d}$ bilayer penta-silicene, the crystal possesses pb2b or pm2a layer group symmetry which belongs to the orthorhombic crystal systems, and the independent elastic constants are: $C_{11}=C_{22}=63.83 \mathrm{~N} / \mathrm{m}, C_{12}=26.92 \mathrm{~N} / \mathrm{m}$ and $C_{66}=50.43 \mathrm{~N} / \mathrm{m}$. For mechanical stability, the following criteria must be fulfilled for 2D tetragonal systems:

$$
C_{11}>\left|C_{12}\right|, C_{66}>0
$$

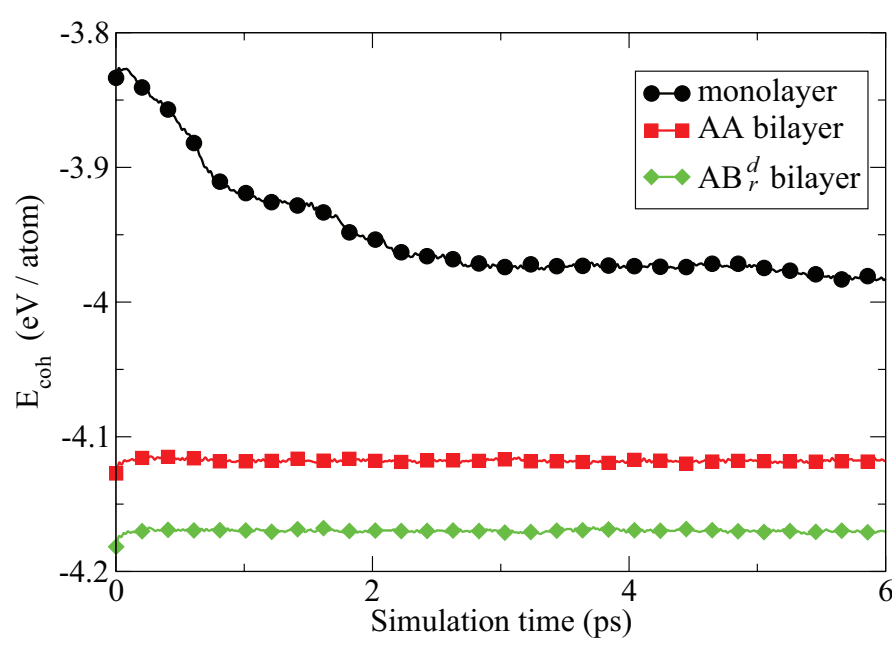

Fig. 5 The cohesive energy of monolayer and $\mathrm{AA}$ and $\mathrm{AB}_{r}^{d}$ stacked bilayer penta-silicene as a function of time at a temperature of $100 \mathrm{~K}$ under NpT-ensemble.

while 2D orthorhombic systems should satisfy:

$$
C_{11}>0, C_{11} C_{22}>C_{12}^{2}, C_{66}>0 .
$$

As one can see, these criteria are satisfied by $\mathrm{AA}$ and $\mathrm{AB}_{r}^{d}$ bilayer penta-silicene which ensures their mechanical stability. Additionally, in Table 3, we list the (2D) Young's modulus, shear modulus and Poisson's ratio of bilayer penta-silicene systems. An interesting aspect of the possion's ratio of $\mathrm{AB}_{r}^{d}$ is that it is quite high and close to the theoretical limit of 0.5 . This means that this $2 \mathrm{D}$ material, prefers to change its shape rather than its surface area under strain, similar to the (3D) cases of rubber and water.

Table 3 Mechanical properties of $\mathrm{AB}_{r}^{d}$ bilayer penta-silicene

\begin{tabular}{lccc}
\hline Stacking & $E[N / m]$ & $G[N / m]$ & $v$ \\
\hline $\mathrm{AA}$ & 88.40 & 39.53 & 0.36 \\
$\mathrm{AB}_{r}{ }_{r}$ & 52.47 & 50.41 & 0.42 \\
\hline
\end{tabular}

$4 \mid$ 1-7 


\subsection{Relative phase stability}

In this section we compare the cohesive energy of bilayer pentasilicene to the more familiar bilayer hexagonal silicene structures (h-Si). We examined 3 different stacking types for h-Si bilayers, denoted as h-Si1, h-Si2, and h-Si3. To the best of our knowledge, these are the most stable hexagonal bilayer structures of silicene predicted so far. The h-Si2 structure corresponds to the re-DLSi structure suggested by Morishita et al. ${ }^{34}$ and the h-Si3 is the hex-OR- $2 \times 2$ structure that was recently proposed by Sakai and Oshiyama ${ }^{35}$. These structures are constructed from the structure information provided by the authors in the supplementary material of the corresponding papers and re-optimized with our computational procedure. The h-Sil is a new stable bilayer h-Si structure that we discovered (see ESI $\dagger$ for details). It is composed of two planar, non-buckled, compressed hexagonal silicene planes that are shifted along the crystal plane. This structure is interesting because although its cohesive energy is close to the former two cases, it has a non-buckled nature. To the best of our knowledge, it is the most stable non-buckled bilayer silicene discovered so far.

The cohesive energies with and without vdW corrections of all the stable bilayer Si systems are given in Table 4. It is seen that the $\mathrm{AB}_{r}^{d}$ bilayer penta-silicene system has the lowest energy, about $10 \mathrm{meV} /$ atom ( $28 \mathrm{meV} /$ atom with vdW) less than the most stable hexagonal silicene bilayer h-Si3. This means that the $\mathrm{AB}_{r}^{d}$ penta-silicene structure is the most stable bilayer silicon structure predicted so far, which is a very surprising result. The AA-stacked penta-silicene has slightly higher energy than h-Si2 and h-Si3.

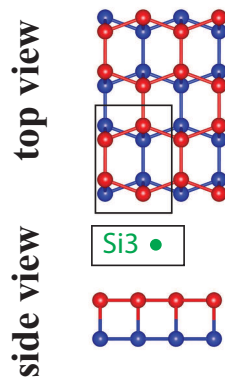

(1) h-Si1

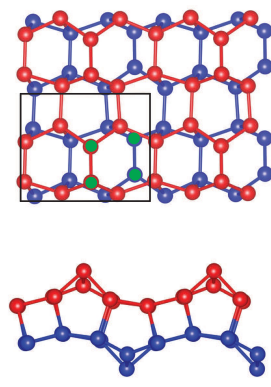

(2) h-Si2

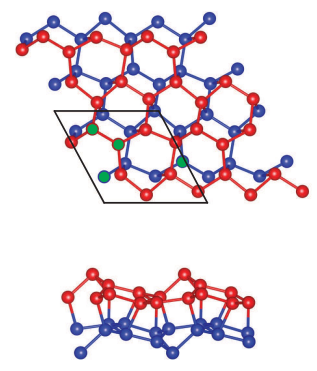

(3) h-Si3
Fig. 6 Top and side views of the atomic structures of the $2 \times 2$ supercell of the three examined hexagonal silicene bilayers.

Table 4 The interlayer distance $d_{\text {inter }}$, the nearest-neighbor bond length range $\left(d_{\min / \max }\right)$ and the cohesive energy per atom with $\left(E_{c o h}^{v d W}\right)$ and without $\left(E_{c o h}\right)$ vdW correction of the most stable hexagonal bilayer silicene and bilayer penta-silicene.

\begin{tabular}{lccccc}
\hline structure & $\begin{array}{c}\mathrm{d}_{\text {inter }} \\
(\AA)\end{array}$ & $\begin{array}{c}\mathrm{d}_{\min } \\
(\AA)\end{array}$ & $\begin{array}{c}\mathrm{d}_{\max } \\
(\AA)\end{array}$ & $\begin{array}{c}\mathrm{E}_{\text {coh }} \\
(\mathrm{eV} / \mathrm{atom})\end{array}$ & $\begin{array}{c}\mathrm{E}_{\text {coh }}^{\text {vdW }} \\
(\mathrm{eV} / \mathrm{atom})\end{array}$ \\
\hline AA bilayer penta-silicene & 0.795 & 2.363 & 2.468 & -4.129 & -4.376 \\
$\mathrm{AB}_{r}^{d}$ bilayer penta-silicene & 1.896 & 2.303 & 2.403 & -4.185 & -4.440 \\
h-Si1 & 2.175 & 2.358 & 2.418 & -4.115 & -4.332 \\
h-Si2 & 1.579 & 2.298 & 2.453 & -4.165 & -4.403 \\
h-Si3 & 1.378 & 2.288 & 2.473 & -4.175 & -4.412 \\
\hline
\end{tabular}
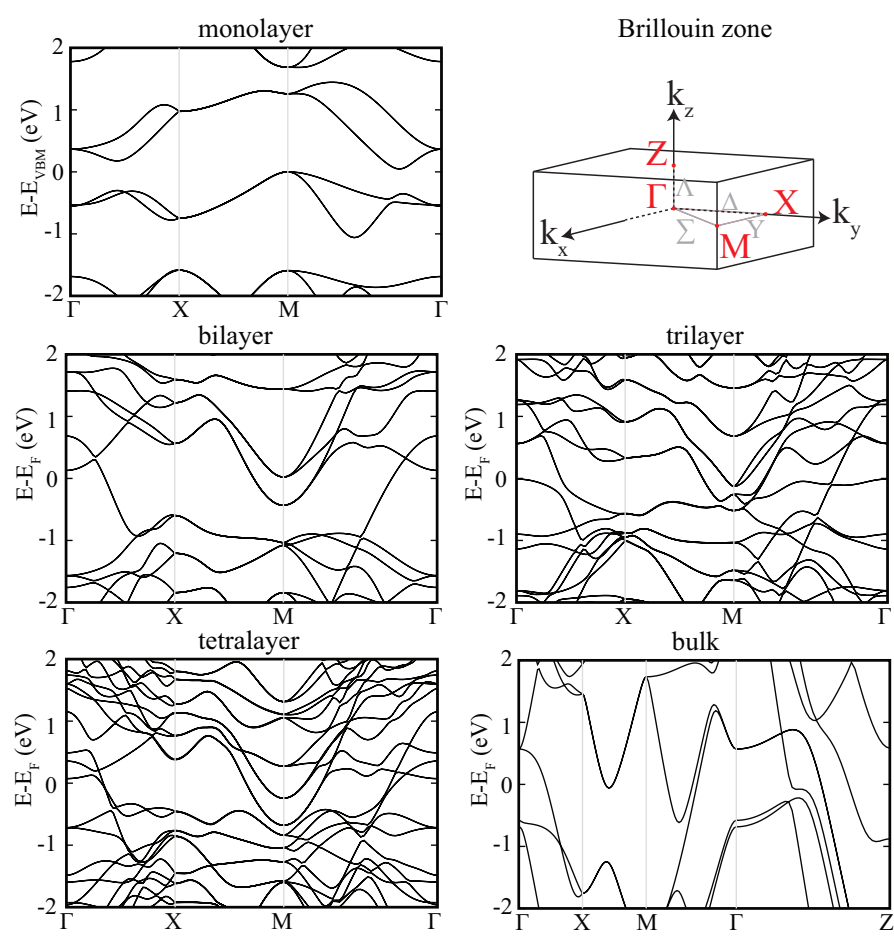

Fig. 7 Electronic band structure of AA stacked few-layer and bulk pentasilicene, and a schematic of the first Brillouin zone.

\subsection{Electronic properties}

In the last part of this work, we investigate the electronic properties of few-layer and bulk penta-silicene. These electronic properties are mainly determined by the electronic spectrum. In Fig. 7 and Fig. 8(a), the electronic band structure of respectively AA and $\mathrm{AB}_{r}^{d}$ penta-silicene multilayers and their bulk counterpart is shown. The band structure of the unstable monolayer is also calculated for comparison. Monolayer penta-silicene is an indirect semiconductor with a band gap of $0.046 \mathrm{eV}$ (PBE). The band-edge states are mainly composed of $p_{z}$ orbitals of $\mathrm{Si} 3$ atoms. In contrast to this, all AA-stacked multilayers are metallic. In the case of the $\mathrm{AB}_{r}^{d}$ structure, the semiconducting properties of monolayer penta-silicene are preserved, but the band gap changes somewhat with the number of layers. This can be understood from the position of the electron and hole states which correspond to the conduction band minimum (CBM) and the valence band maximum (VBM), respectively. As seen in Fig. 8(a), the VBM and CBM states are always localized on the outermost layers. In other words, the electronic properties are mainly determined by the surface region which is nearly independent of the slab thickness. For $\mathrm{AB}_{r}$-stacked bulk penta-silicene, there is no surface and the VBM and CBM correspond to bulk states. This explains the much larger band gap $(1.33 \mathrm{eV})$ in the bulk case.

Strain is an effective tool to modulate the electronic properties of $2 \mathrm{D}$ semiconductors. Here we investigate the band gap variation of $\mathrm{AB}_{r}^{d}$ bilayer penta-silicene with respect to both uniaxial and biaxial tensile strain up to $10 \%$, see Fig. 9(a). We observe, in both cases, a small kink in the band gaps that gives rise to nonmonotonic behavior. For uniaxial strain, the band gap first increases from $0.12 \mathrm{eV}$ to $0.27 \mathrm{eV}$ at $4 \%$ strain, and then decreases 

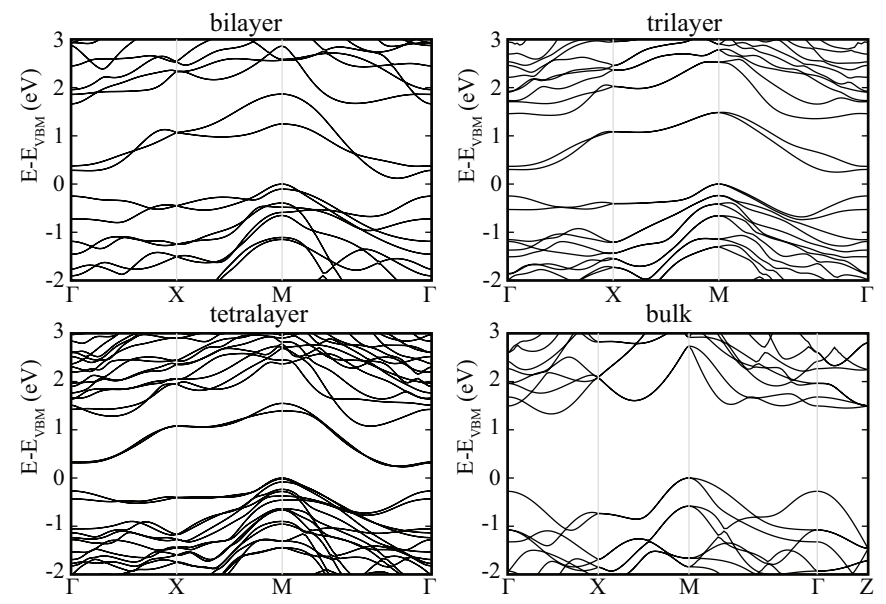

(a) Electronic band structures

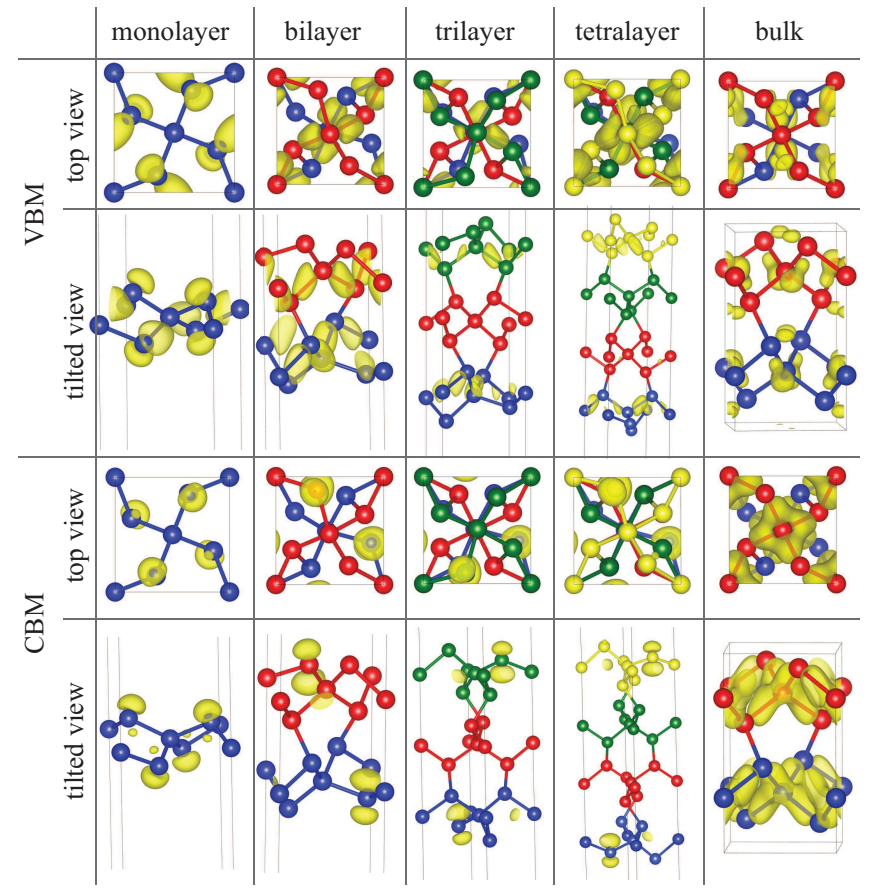

(b) Charge density

Fig. 8 (a) Electronic band structure of $\mathrm{AB}_{r}^{d}$ stacked few-layer and $\mathrm{AB}_{r}$ stacked bulk penta-silicene, and (b) the corresponding charge distribution of CBM and VBM. The results for the monolayer are shown for comparison. again until the system becomes metallic at 10\%. A similar behavior is found for biaxial strain where the band gap increases up to $0.25 \mathrm{eV}$ at $2 \%$ strain and the system becomes metallic at $6 \%$ tensile strain. In order to explain the kink in the band gap evolution, we plot the relevant band edges with respect to the vacuum level as a function of strain. In the figure, the high-symmetry points of the Brillouin zone where the edges are located are given between round brackets, while the notation VBM-1 is used to denote the first band below the VBM (in the band structure without strain). As can be seen from Fig. 9(b) and (c), while the $\mathrm{CBM}(\Sigma)$ s slightly decrease in a monotonic way, the $\mathrm{VBM}(\mathrm{M})$ crosses the VBM-1(M) once under uniaxial strain, and two crosses, first the $\operatorname{VBM}(\mathrm{M})$ switches its position from $\mathrm{M}$ to $\mathrm{Y}$, and then back to $\mathrm{M}$ but for a lower band, occur for biaxial strain. These crosses give rise to abrupt changes in the band gaps.
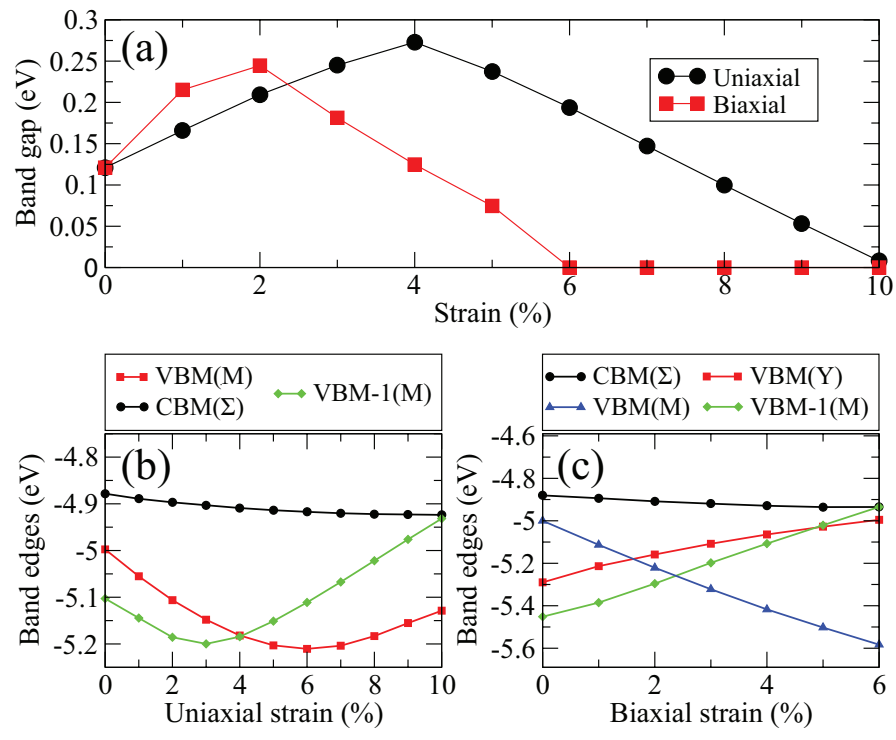

Fig. 9 Band gaps (a) and band edges for uniaxially (b) and biaxially (c) strained $\mathrm{AB}_{r}^{d}$ bilayer penta-silicene.

\section{Summary and Conclusions}

In this work, we proposed several stable structures for few-layer penta-silicene. The stability of these structures was confirmed via their phonon spectrum, finite-temperature molecular dynamics, and their mechanical properties. The type of stacking mode, AA or $\mathrm{AB}$, of few-layer penta-silicene has a crucial influence on the electronic properties: AA-stacked systems are metallic, while $\mathrm{AB}_{r}^{d}$ stacked ones are semiconducting . Surprisingly, the $\mathrm{AB}_{r}^{d}$ stacked bilayer penta-silicene has lower energy than the most stable bilayer hexagonal silicene structures, which makes it the most stable predicted form of bilayer silicon. We found that it is possible to engineer the electronic properties by tensile strain. Both quantitative and qualitative changes can be induced in this way. We explained the variation of the band gap from the band edge crossings that occur under strain. In conclusion, the proposed bilayer penta-silicene structure is the most stable form of bilayer silicon predicted so far and it was shown to have interesting tunable properties. 


\section{Acknowledgments}

This work was supported by the Fonds Wetenschappelijk Onderzoek (FWO-Vl). The computational resources used in this work were provided by the VSC (Flemish Supercomputer Center), funded by the Hercules Foundation and the Flemish Governmentdepartment EWI.

\section{References}

1 K. S. Novoselov, A. K. Geim, S. V. Morozov, D. Jiang, Y. Zhang, S. V. Dubonos, I. V. Grigorieva and A. A. Firsov, Science, 2004, 306, 666-669.

2 K. S. Novoselov, D. Jiang, F. Schedin, T. J. Booth, V. V. Khotkevich, S. V. Morozov and A. K. Geim, PNAS, 2005, 102, 1045110453.

3 A. K. Geim and K. S. Novoselov, Nat Mater, 2007, 6, 183-191.

4 F. Schedin, A. K. Geim, S. V. Morozov, E. W. Hill, P. Blake, M. I. Katsnelson and K. S. Novoselov, Nat Mater, 2007, 6, 652-655.

5 X. Wang, L. Zhi and K. Müllen, Nano Letters, 2008, 8, 323327.

6 K. S. Novoselov, A. K. Geim, S. V. Morozov, D. Jiang, M. I. Katsnelson, I. V. Grigorieva, S. V. Dubonos and A. A. Firsov, Nature, 2005, 438, 197-200.

7 K. Bolotin, K. Sikes, Z. Jiang, M. Klima, G. Fudenberg, J. Hone, P. Kim and H. Stormer, Solid State Commun., 2008, 146, $351-355$.

8 C. Lee, X. Wei, J. W. Kysar and J. Hone, Science, 2008, 321, 385-388.

9 M. Xu, T. Liang, M. Shi and H. Chen, Chemical Reviews, 2013, 113, 3766-3798.

10 G. R. Bhimanapati, Z. Lin, V. Meunier, Y. Jung, J. Cha, S. Das, D. Xiao, Y. Son, M. S. Strano, V. R. Cooper, L. Liang, S. G. Louie, E. Ringe, W. Zhou, S. S. Kim, R. R. Naik, B. G. Sumpter, H. Terrones, F. Xia, Y. Wang, J. Zhu, D. Akinwande, N. Alem, J. A. Schuller, R. E. Schaak, M. Terrones and J. A. Robinson, ACS Nano, 2015, 9, 11509-11539.

11 S. Z. Butler, S. M. Hollen, L. Cao, Y. Cui, J. A. Gupta, H. R. Gutiérrez, T. F. Heinz, S. S. Hong, J. Huang, A. F. Ismach, E. Johnston-Halperin, M. Kuno, V. V. Plashnitsa, R. D. Robinson, R. S. Ruoff, S. Salahuddin, J. Shan, L. Shi, M. G. Spencer, M. Terrones, W. Windl and J. E. Goldberger, ACS Nano, 2013, 7, 2898-2926.

12 S. Zhang, J. Zhou, Q. Wang, X. Chen, Y. Kawazoe and P. Jena,
PNAS, 2015, 112, 2372-2377.

13 Y. Ding and Y. Wang, J. Mater. Chem. C, 2015, 3, 1134111348.

14 X. Li, Y. Dai, M. Li, W. Wei and B. Huang, J. Mater. Chem. A, 2015, 3, 24055-24063.

15 G. Kresse and J. Hafner, Phys. Rev. B, 1993, 47, 558-561.

16 G. Kresse and J. Hafner, Phys. Rev. B, 1994, 49, 14251-14269.

17 G. Kresse and J. Furthmüller, Comput. Mater. Sci., 1996, 6, 15 -50 .

18 G. Kresse and J. Furthmüller, Phys. Rev. B, 1996, 54, 1116911186.

19 P. E. Blöchl, Phys. Rev. B, 1994, 50, 17953-17979.

20 G. Kresse and D. Joubert, Phys. Rev. B, 1999, 59, 1758-1775.

21 J. P. Perdew, K. Burke and M. Ernzerhof, Phys. Rev. Lett., 1996, 77, 3865-3868.

22 J. P. Perdew, K. Burke and M. Ernzerhof, Phys. Rev. Lett., 1997, 78, 1396-1396.

23 S. Grimme, J. Antony, S. Ehrlich and H. Krieg, The Journal of Chemical Physics, 2010, 132,.

24 S. Grimme, S. Ehrlich and L. Goerigk, Journal of Computational Chemistry, 2011, 32, 1456-1465.

25 A. Togo and I. Tanaka, Scr. Mater., 2015, 108, 1 - 5.

26 M. Parrinello and A. Rahman, Phys. Rev. Lett., 1980, 45, 1196-1199.

27 M. Parrinello and A. Rahman, J. Appl. Phys., 1981, 52, 71827190.

28 M. Allen and D. Tildesley, Computer Simulation of Liquids, Clarendon Press, 1989.

29 V_Sim atomic visualization package available online, http://inac.cea.fr/L_Sim/V_Sim/index.en.html, Accessed: 2016-03-25.

30 K. Momma and F. Izumi, J. Appl. Cryst., 2011, 44, 1272-1276.

31 Z. Wang, F. Dong, B. Shen, R. Zhang, Y. Zheng, L. Chen, S. Wang, C. Wang, K. Ho, Y.-J. Fan, B.-Y. Jin and W.-S. Su, Carbon, 2016, 101, $77-85$.

32 Z. Zhao, F. Tian, X. Dong, Q. Li, Q. Wang, H. Wang, X. Zhong, B. Xu, D. Yu, J. He, H.-T. Wang, Y. Ma and Y. Tian, JACS, 2012, 134, 12362-12365.

33 F. Mouhat and F.-X. Coudert, Phys. Rev. B, 2014, 90, 224104.

34 T. Morishita, M. J. Spencer, S. P. Russo, I. K. Snook and M. Mikami, Chem. Phys. Lett., 2011, 506, 221-225.

35 Y. Sakai and A. Oshiyama, Phys. Rev. B, 2015, 91, 201405. 